\title{
Frontal Lobe Atrophy in Depression after Stroke
}

\author{
W. K. Tang, ${ }^{1,2}$ Y. K. Chen, ${ }^{1,3}$ J. Y. Lu, ${ }^{1}$ V. C. T. Mok, ${ }^{4}$ Winnie C. W. Chu, ${ }^{5}$ \\ Gabor S. Ungvari, ${ }^{6,7}$ and K. S. Wong ${ }^{4}$ \\ ${ }^{1}$ Department of Psychiatry, Chinese University of Hong Kong, Hong Kong \\ ${ }^{2}$ Department of Psychiatry, Shatin Hospital, Hong Kong \\ ${ }^{3}$ Department of Neurology, Dongguan People's Hospital, Dongguan, Guangdong, China \\ ${ }^{4}$ Department of Medicine and Therapeutics, Chinese University of Hong Kong, Hong Kong \\ ${ }^{5}$ Department of Imaging \& Interventional Radiology, Chinese University of Hong Kong, Hong Kong \\ ${ }^{6}$ School of Psychiatry \& Clinical Neurosciences, University of Western Australia, Perth, Western Australia, Australia \\ ${ }^{7}$ University of Notre Dame Australia/Marian Centre, Perth, Western Australia, Australia
}

Correspondence should be addressed to W. K. Tang; tangwk@cuhk.edu.hk

Received 27 June 2012; Accepted 21 January 2013

Academic Editor: Helmuth Steinmetz

Copyright (C) 2013 W. K. Tang et al. This is an open access article distributed under the Creative Commons Attribution License, which permits unrestricted use, distribution, and reproduction in any medium, provided the original work is properly cited.

Background. Frontal lobe atrophy (FLA) is associated with late life depression. However, the role that FLA plays in the development of depression after stroke (DAS) remains unknown. This study thus examined the association between FLA and DAS. Methods. A convenience sample of 705 Chinese patients with acute ischemic stroke admitted to the acute stroke unit of a university-affiliated regional hospital in Hong Kong participated in the study. A psychiatrist administered the Structural Clinical Interview for DSMIV to all patients and made a diagnosis of DAS three months after the index stroke. Results. Eighty-five (12.1\%) patients were diagnosed with DAS. In univariate analysis, the DAS patients were more likely to have severe FLA (14.1\% versus 5.6\%). Severe FLA remained an independent predictor of DAS in multivariate analysis, with an odds ratio of 2.6 (95\% confidence intervals $=1.2-5.9)$. Conclusions. The results suggest that FLA may play a role in the pathogenesis of DAS, which supports the hypothesis that cumulative vascular burden may be important in predicting DAS. Further investigations are needed to clarify the impact of FLA on the clinical presentation, treatment response, and outcome of DAS in stroke survivors.

\section{Introduction}

Depression is the most common psychiatric disorder following stroke. Depression is associated with excessive disability, cognitive impairment, and mortality [? ]. The neuroanatomical model of depression after stroke (DAS), a depressive illness in patients with well-established cerebrovascular disease, remains unclear. There is no compelling evidence to support the hypothesis of a close relationship between lesion location and DAS [? ], although it has been suggested recently that chronic vascular burden may be an important factor in the development of DAS [?].

The frontal lobes are thought to be involved in the pathogenesis of depression [?]. Frontal lobe atrophy (FLA) is associated with late life depression [?] and depression occurring in multiple sclerosis [?]. FLA in stroke can be conceptualised as a sign of vascular damage to brain structures [?], and as such it contributes to the development of vascular depression [? ]. To date, no study has found an association between FLA and DAS [? ? ? ? ? ]. The aim of this study was thus to reexamine the relationship between FLA and DAS in stroke survivors.

\section{Materials and Methods}

2.1. Participants. A total of 3,219 patients with first-ever or recurrent acute ischemic stroke were admitted to the Acute Stroke Unit of the Prince of Wales Hospital (PWH) between June 2004 and July 2008. The PWH is a university-affiliated general hospital serving a population of 800,000 in Hong Kong. Of the 1,353 patients who received an MRI examination, a convenience sample of 705 (52.1\%) was recruited 
for this study. Compared to the participants, the excluded patients $(n=648)$ were older $(69.0 \pm 11.4$ versus $64.4 \pm 11.4$, $P<0.001)$, less likely to be female (53.5\% versus $59.1 \%$, $P=0.038)$, and more likely to have a higher National Institute of Health Stroke Scale (NIHSS) score (6.9 \pm 5.8 versus $4.4 \pm 3.4$, $P<0.001)$.

The inclusion criteria for the study were (1) Chinese ethnicity, (2) Cantonese as the primary language, (3) aged 18 or above, (4) well-documented (clinical presentation and CT scan of the brain) first or recurrent acute stroke occurring within the seven days prior to admission, and (5) ability and willingness to give consent. The exclusion criteria were (1) transient ischemic attack, cerebral haemorrhage, subdural haematoma, or subarachnoid haemorrhage, (2) history of a central nervous system disease such as tumour, trauma, hydrocephalus, and Parkinson's disease, (3) aphasia, defined as a score of 2 or more in the best language item of the NIHSS [?], and (4) moderate to severe cognitive impairment, defined as a Mini-Mental State Examination (MMSE) [? ] score less than 17.

The study protocol was approved by the Clinical Research Ethics Committee of the Chinese University of Hong Kong. All participants signed a consent form.

2.2. Collection of Demographic and Clinical Data. A research nurse, who was blind to the psychiatric diagnoses, collected the demographic data (age, sex, and education level in terms of school years) and assessed stroke severity using the NIHSS within two days of admission. A research assistant evaluated all participants with the MMSE and the Lubben Social Network Scale (LSNS) [? ] three months after the onset of the index stroke. The LSNS is a composite social network scale that was specifically designed for use in the elderly. It measures the level of social support that patients receive and their social interactions with relatives and friends. The LSNS has been translated into Chinese and validated amongst the Hong Kong elderly [? ]. Exposure to recent life events was assessed with the Modified Life Event Scale (MLES) [? ?].

2.3. Assessment of DAS. Three months after the onset of the index stroke, a psychiatrist, who was blind to the participants' radiological data, administered the Chinese version of the Structured Clinical Interview for DSM-IV [? ? ] at a research clinic. The timing of the assessment was chosen to avoid the period of transient emotional adjustment to the disability caused by stroke [?].

2.4. Radiological Examination. MRI with diffusion-weighted imaging (DWI) and proton density sequences was performed on each participant with a 1.5-T system (Sonata, Siemens Medical, Erlangen, Germany) within seven days of admission.

DWI spin EPI $($ TR/TE/excitation $=180 / 122 / 4$, matrix $=128 \times 128, \mathrm{FOV}=230 \mathrm{~mm}$, slice thickness $/$ gap $=$ $5 \mathrm{~mm} / 1 \mathrm{~mm}$, EPI factor $=90$, and acquisition time $=55$ seconds) with three orthogonally applied gradients was used with values of 1000 and 500. Axial gradient echo images were acquired as the second sequence, with imaging parameters of
TR/TE/excitation $=350 / 30 / 2$, a flip angle of 30 degrees, slice thickness $/$ gap $=5 \mathrm{~mm} / 0.5 \mathrm{~mm}, \mathrm{FOV}=230 \mathrm{~mm}$, matrix $=$ $256 \times 256$, and acquisition time $=5$ minutes and 4 seconds. Axial SE T1 (TR/TE/excitation $=425 / 14 / 2, \mathrm{FOV}=230 \mathrm{~mm}$, slice thickness $/$ gap $=5 \mathrm{~mm} / 0.5 \mathrm{~mm}$, matrix $=256 \times 256$, and acquisition time $=4$ minutes, 28 seconds) and TSE T2 $(\mathrm{TR} / \mathrm{TE} /$ excitation $=2500 / 120 / 1$, turbo factor $=15, \mathrm{FOV}=$ $230 \mathrm{~mm}$, slice thickness/gap $=5 \mathrm{~mm} / 0.5 \mathrm{~mm}$, matrix $=$ $256 \times 256$, and acquisition time $=1$ minute, 39 seconds) images were also acquired.

A neurologist, who was blind to the psychiatric diagnoses, assessed the MRIs to determine the following.

(1) Brain Atrophy. Brain atrophy was quantified with a three-point visual cortical atrophy scoring method [?] to rate the severity of cortical atrophy on the T1-weighted images. This method provides two standard images that represent mild and moderate lobar atrophy severity. Severe lobar atrophy was recorded when the extent of atrophy on either side of the lobe was more severe than that on the second standard image. Atrophy in the frontal, parietal, temporal and occipital lobes was rated separately. The intrarater reliability of the lobar atrophy measurement, which was tested on 20 cases, was good (the weighted kappa values for the frontal, parietal, and temporal lobes were $0.90,0.81$, and 0.71 , resp.).

(2) Brain Infarcts. The number and volume of acute infarcts were recorded. The total area of acute infarcts on the DWI was measured with manual outlines. Restricted water diffusion was identified on diffusionweighted images with $b$ values of 1000 . The total volume was calculated by multiplying the total area by the sum of the slice thickness and the gap. Intrarater reliability tests were performed on 20 participants, yielding kappa values of 0.96 and 0.94 for the volume and number of infarcts, respectively.

(3) White Matter Hyperintensities (WMHs). The severity of WMHs was graded using a 4-point scale [? ? ]. Periventricular white matter (PVWMHs) and deep white matter (DWMHs) hyperintensities were scored on axial proton density images. The intrarater agreement was good for both of the PVWMH (weighted kappa: 0.87) and DWMH ratings (weighted kappa: 0.93).

2.5. Statistical Analysis. All statistical tests were performed using SPSS for Windows (Release 14.0, SPSS Inc., Chicago, IL, USA). The demographic and clinical variables (age, sex, education level, and NIHSS and MMSE scores) and radiological characteristics of the participants with DAS were compared with those of the non-DASs group using the $\chi^{2}$ test, Student's $t$-test, and Mann-Whitney $U$ test, as appropriate. Risk factors with a value of $P<0.10$ were then analysed via multivariate logistic regression using a forward stepwise selection strategy. If the correlations between any of the putative risk factors were $\geq 0.50$, then additional models were examined to rule out collinearity. The odds ratio of any independent risk factor 
TABLE 1: Univariate and multivariate analyses of the clinical and radiological determinants of DAS.

\begin{tabular}{lccc}
\hline & $\begin{array}{c}\text { DAS } \\
\text { Mean } \pm \text { SD }\end{array}$ & $\begin{array}{c}\text { No DAS } \\
\text { Mean } \pm \text { SD }\end{array}$ & $P^{*}$ value \\
\hline$N$ & 85 & 620 & \\
Age & $66.9 \pm 11.6$ & $66.2 \pm 11.6$ & $0.632^{\dagger}$ \\
Female & $50(58.8)$ & $238(38.4)$ & $<0.001^{\ddagger}$ \\
Education (years) & $4.4 \pm 4.7$ & $5.7 \pm 4.7$ & 0.005 \\
Previous stroke & $23(27.1)$ & $123(19.8)$ & $0.123^{\ddagger}$ \\
NIHSS during index & $5.1 \pm 3.5$ & $4.4 \pm 3.4$ & 0.029 \\
admission & $24.8 \pm 3.6$ & $26.1 \pm 3.2$ & 0.002 \\
MMSE & $25.5 \pm 8.4$ & $30.4 \pm 8.2$ & $<0.001^{\dagger}$ \\
LSNS & $2.3 \pm 1.2$ & $1.7 \pm 0.8$ & $<0.001$ \\
MLES & $14(16.5)$ & $7(1.1)$ & $<0.001^{\ddagger}$ \\
$\begin{array}{l}\text { Past history of depression } \\
\text { Severe brain atrophy }\end{array}$ & $12(14.1)$ & $35(5.6)$ & $0.003^{\ddagger}$ \\
$\quad \begin{array}{l}\text { Frontal lobe } \\
\text { Temporal lobe }\end{array}$ & $6(7.1)$ & $76(12.3)$ & $0.161^{\ddagger}$ \\
$\quad \begin{array}{l}\text { Parietal lobe } \\
\text { Occipital lobe }\end{array}$ & $14(16.5)$ & $97(15.6)$ & $0.845^{\ddagger}$ \\
$\begin{array}{l}\text { Periventricular } \\
\text { hyperintensities }\end{array}$ & $13(15.3)$ & $95(15.3)$ & $1.000^{\ddagger}$ \\
$\begin{array}{l}\text { Deep white matter } \\
\text { hyperintensities }\end{array}$ & $1.2 \pm 0.8$ & $1.3 \pm 0.9$ & 0.653 \\
$\begin{array}{l}\text { Number of acute infarcts } \\
\text { Acute infarct volume }\end{array}$ & $1.0 \pm 1.4$ & $1.3 \pm 1.1$ & 0.131 \\
\hline (mm & $2.4 \pm 5.2$ & $2.9 \pm 9.1$ & 0.772 \\
\hline
\end{tabular}

NIHSS: National Institute of Health Stroke Scale; MMSE: Mini-Mental State Examination; LSNS: Lubben Social Network Scale; MLES: Modified Life Event Scale; SD: standard deviation.

${ }^{*}$ In comparison to the DAS group by means of Mann-Whitney $U$ test unless otherwise specified.

${ }^{\dagger}$ In comparison to the DAS group by means of $t$-test.

${ }^{\ddagger}$ In comparison to the DAS group by means of Chi-square $U$ test.

was interpreted as the risk of subsequent DASs when all other risk factors were held constant. The level of significance was set at 0.05 .

\section{Results}

Eighty-five patients were diagnosed with DAS. The participants' demographic, clinical, and MRI characteristics stratified by DAS status are shown in Table 1 .

Severe FLA was more common in the DAS group. Participants with DAS were also more likely to be female and to have a history of depression, a lower level of education, and higher NIHSS and MLES scores but lower MMSE and LSNS scores.

The following variables were entered into the regression model: sex, education, severe FLA, history of depression, and the NIHSS, MMSE, MLES, and LSNS scores. Severe FLA was a significant independent predictor of DAS, with an odds ratio of $2.6(P=0.016)$ (Table 2$)$. Severe FLA remained a significant predictor of DAS in additional regression models
TABLE 2: Multivariate logistic model of the clinical and radiological determinants of DAS.

\begin{tabular}{lcc}
\hline Variable & Odds ratio $(95 \% \mathrm{CI})$ & $P$ value \\
\hline Severe frontal lobe atrophy & $2.648(1.198-5.856)$ & 0.016 \\
History of depression & $11.99(3.953-36.372)$ & $<0.001$ \\
MLES & $1.925(1.490-2.487)$ & $<0.001$ \\
LSNS & $0.943(0.915-0.972)$ & $<0.001$ \\
NIHSS & $1.106(1.032-1.185)$ & 0.004 \\
Female & $2.312(1.370-3.902)$ & 0.002 \\
MMSE & - & $\mathrm{NS}$ \\
Education & - & $\mathrm{NS}$ \\
\hline$R^{2}=0.259$. & &
\end{tabular}

$R^{2}=0.259$

NIHSS: National Institute of Health Stroke Scale; MMSE: Mini-Mental State Examination; LSNS: Lubben Social Network Scale; MLES: Modified Life Event Scale; NS: nonsignificant.

(data not shown), in which either age was entered into the regression (odds ratio $=3.00, P=0.007$ ) or patients with prior depression were excluded (odds ratio $=2.5, P=0.026$ ).

\section{Discussion}

To the best of our knowledge, this is the first MRI study to show that severe FLA is an independent predictor of DAS.

Although a number of studies have examined the relationship between brain atrophy and DAS, none has focused on the frontal lobes. In addition, only broad measures of atrophy, such as global [?], cortical [? ? ], subcortical [? ? ], and central atrophy, [? ? ] or ventricle-to-brain ratio were analysed in these studies [?], which may explain their negative findings.

FLA in stroke can be the result of degeneration and/or ischemic injuries. Brain atrophy is associated with subcortical white matter lesions both in patients with vascular dementia [?] and in healthy controls [? ]. FLA in stroke is also associated with white matter lesions and small vessel disease [? ]. Our findings support the recent hypothesis that DAS is related to cumulative vascular brain pathology rather than a single infarct [? ]. Another indicator of chronic vascular burden, namely, lacunae, is also associated with DAS [?].

One limitation of this study is that it was conducted in a hospital-based sample, and thus its findings may not be applicable to patients treated in other settings. In addition, the exclusion rate was high which might have resulted in a biased sample. Furthermore, the number of cases of DAS with FLA was small and a large number of possible confounding variables were adjusted in the multivariate analysis; hence the findings should be regarded as tentative and need replication. Finally, FLA was only one of the six significant factors in the final regression model.

\section{Conclusions}

In conclusion, the results of this study indicate that there is an association between FLA and DAS. Further investigations are needed to clarify the impact of FLA on the clinical 
presentation, treatment response, and outcome of DAS in stroke survivors.

\section{Conflict of Interests}

The authors report no conflict of interests.

\section{Acknowledgment}

This work was supported by the Research Grant Council of the HKSAR.

\section{References}

[1] E. M. Whyte and B. H. Mulsant, "Post stroke depression: epidemiology, pathophysiology, and biological treatment," Biological Psychiatry, vol. 52, no. 3, pp. 253-264, 2002.

[2] A. J. Carson, S. MacHale, K. Allen et al., "Depression after stroke and lesion location: a systematic review," The Lancet, vol. 356, no. 9224, pp. 122-126, 2000.

[3] M. Santos, G. Gold, E. Kövari et al., "Differential impact of lacunes and microvascular lesions on poststroke depression," Stroke, vol. 40, no. 11, pp. 3557-3562, 2009.

[4] M. Koenigs and J. Grafman, "The functional neuroanatomy of depression: distinct roles for ventromedial and dorsolateral prefrontal cortex," Behavioural Brain Research, vol. 201, no. 2, pp. 239-243, 2009.

[5] A. Kumar, W. Bilker, H. Lavretsky, and G. Gottlieb, "Volumetric asymmetries in late-onset mood disorders: an attenuation of frontal asymmetry with depression severity," Psychiatry Research, vol. 100, no. 1, pp. 41-47, 2000.

[6] R. Bakshi, D. Czarnecki, Z. A. Shaikh et al., "Brain MRI lesions and atrophy are related to depression in multiple sclerosis," NeuroReport, vol. 11, no. 6, pp. 1153-1158, 2000.

[7] Y. Chen, X. Chen, W. Xiao, V. C. T. Mok, K. S. Wong, and W. K. Tang, "Frontal lobe atrophy is associated with small vessel disease in ischemic stroke patients," Clinical Neurology and Neurosurgery, vol. 111, no. 10, pp. 852-857, 2009.

[8] R. C. Baldwin and J. O'Brien, "Vascular basis of late-onset depressive disorder," British Journal of Psychiatry, vol. 180, pp. 157-160, 2002.

[9] M. Aström, R. Adolfsson, and K. Asplund, "Major depression in stroke patients: a 3-year longitudinal study," Stroke, vol. 24, no. 7, pp. 976-982, 1993.

[10] M. Herrmann, C. Bartels, M. Schumacher, and C. W. Wallesch, "Poststroke depression: is there a pathoanatomic correlate for depression in the postacute stage of stroke?" Stroke, vol. 26, no. 5, pp. 850-856, 1995.

[11] R. Vataja, T. Pohjasvaara, A. Leppävuori et al., "Magnetic resonance imaging correlates of depression after ischemic stroke," Archives of General Psychiatry, vol. 58, no. 10, pp. 925-931, 2001.

[12] A. Verdelho, H. Hénon, F. Lebert, F. Pasquier, and D. Leys, "Depressive symptoms after stroke and relationship with dementia: a three-year follow-up study," Neurology, vol. 62, no. 6, pp. 905-911, 2004.

[13] R. Vataja, T. Pohjasvaara, R. Mäntylä et al., "Depressionexecutive dysfunction syndrome in stroke patients," The American Journal of Geriatric Psychiatry, vol. 13, no. 2, pp. 99-107, 2005.
[14] L. Snaphaan, S. van der Werf, K. Kanselaar, and F. E. de Leeuw, "Post-stroke depressive symptoms are associated with poststroke characteristics," Cerebrovascular Diseases, vol. 28, no. 6, pp. 551-557, 2009.

[15] T. Brott, H. P. Adams Jr., C. P. Olinger et al., "Measurements of acute cerebral infarction: a clinical examination scale," Stroke, vol. 20, no. 7, pp. 864-870, 1989.

[16] H. F. K. Chiu, H. C. B. Lee, and D. Chung, "Reliability and validity of the cantonese version of the mini-mental state examination: a preliminary study," Hong Kong Journal of Psychiatry, vol. 4, no. 2, pp. 25-28, 1994.

[17] J. E. Lubben, "Assessing social network among elderly populations," Family \& Community Health, vol. 11, no. 3, pp. 42-52, 1988.

[18] I. Chi and K. W. Boey, A Mental Health and Social Support Study of the Old-Old in Hong Kong, Resource Paper Series, Department of Social Work and Social Administration, University of Hong Kong, Hong Kong, China, 1994.

[19] E. S. Paykel, B. A. Prusoff, and E. H. Uhlenhuth, "Scaling of life events," Archives of General Psychiatry, vol. 25, no. 4, pp. 340$347,1971$.

[20] H. F. K. Chiu, L. C. W. Lam, I. Chi et al., "Prevalence of dementia in Chinese elderly in Hong Kong," Neurology, vol. 50, no. 4, pp. 1002-1009, 1998.

[21] American Psychiatric Association, Diagnostic and Statistical Manual of Mental Disorders, American Psychiatric Association, Washington, DC, USA, 4th edition, 1994.

[22] I. W. K. Kam, Development of the bilingual (Chinese/English) SCID-I (Structured Clinical Interview for DSM-IV Axis I disorder): a study of its reliability and validity in an in-patient population [Dissertation], Hong Kong College of Psychiatrist, Hong Kong, China, 2000, Part III Examination of Fellowship.

[23] W. K. Tang, S. S. M. Chan, H. F. K. Chiu et al., "Poststroke depression in Chinese patients: frequency, psychosocial, clinical, and radiological determinants," Journal of Geriatric Psychiatry and Neurology, vol. 18, no. 1, pp. 45-51, 2005.

[24] J. Victoroff, W. J. Mack, S. T. Grafton, S. S. Schreiber, and H. C. Chui, "A method to improve interrater reliability of visual inspection of brain MRI scans in dementia," Neurology, vol. 44, no. 12, pp. 2267-2276, 1994.

[25] F. Fazekas, J. B. Chawluk, and A. Alavi, "MR signal abnormalities at $1.5 \mathrm{~T}$ in Alzheimer's dementia and normal aging," American Journal of Roentgenology, vol. 149, no. 2, pp. 351-356, 1987.

[26] M. J. Firbank, J. T. O’Brien, S. Pakrasi et al., "White matter hyperintensities and depression-preliminary results from the LADIS study," International Journal of Geriatric Psychiatry, vol. 20, no. 7, pp. 674-679, 2005.

[27] G. Gainotti, A. Acciarri, A. Bizzarro et al., "The role of brain infarcts and hippocampal atrophy in subcortical ischaemic vascular dementia," Neurological Sciences, vol. 25, no. 4, pp. 192197, 2004.

[28] W. Wen and P. Sachdev, "The topography of white matter hyperintensities on brain MRI in healthy 60- to 64-year-old individuals," NeuroImage, vol. 22, no. 1, pp. 144-154, 2004. 


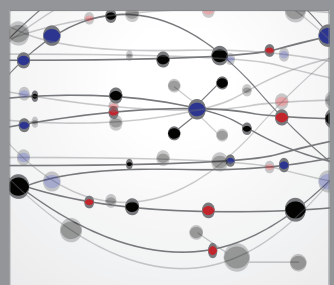

The Scientific World Journal
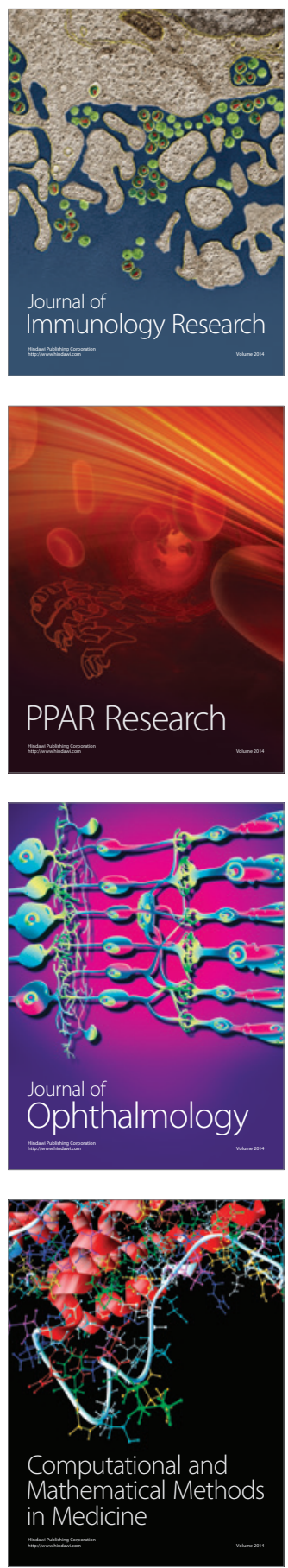

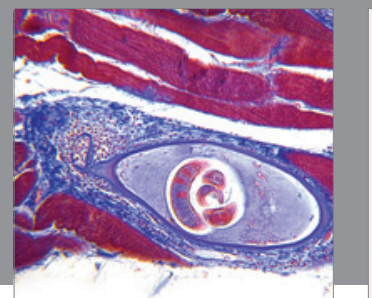

Gastroenterology

Research and Practice
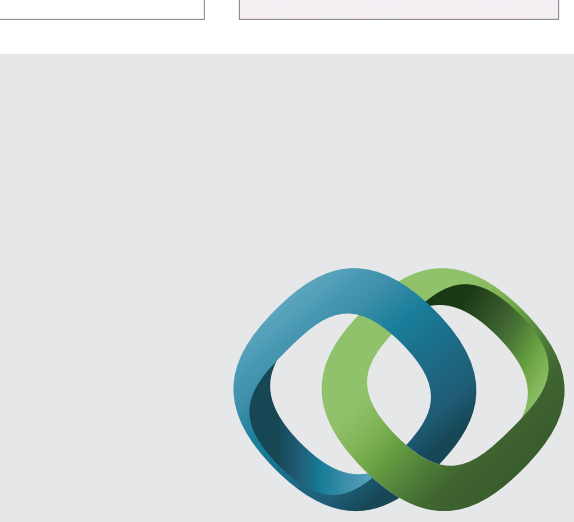

\section{Hindawi}

Submit your manuscripts at

http://www.hindawi.com
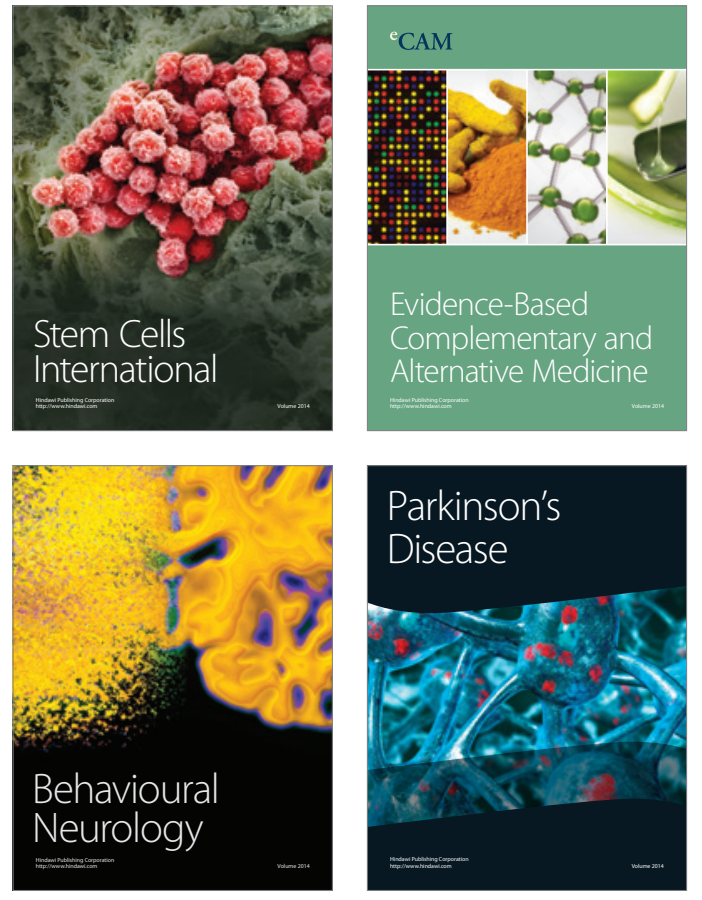
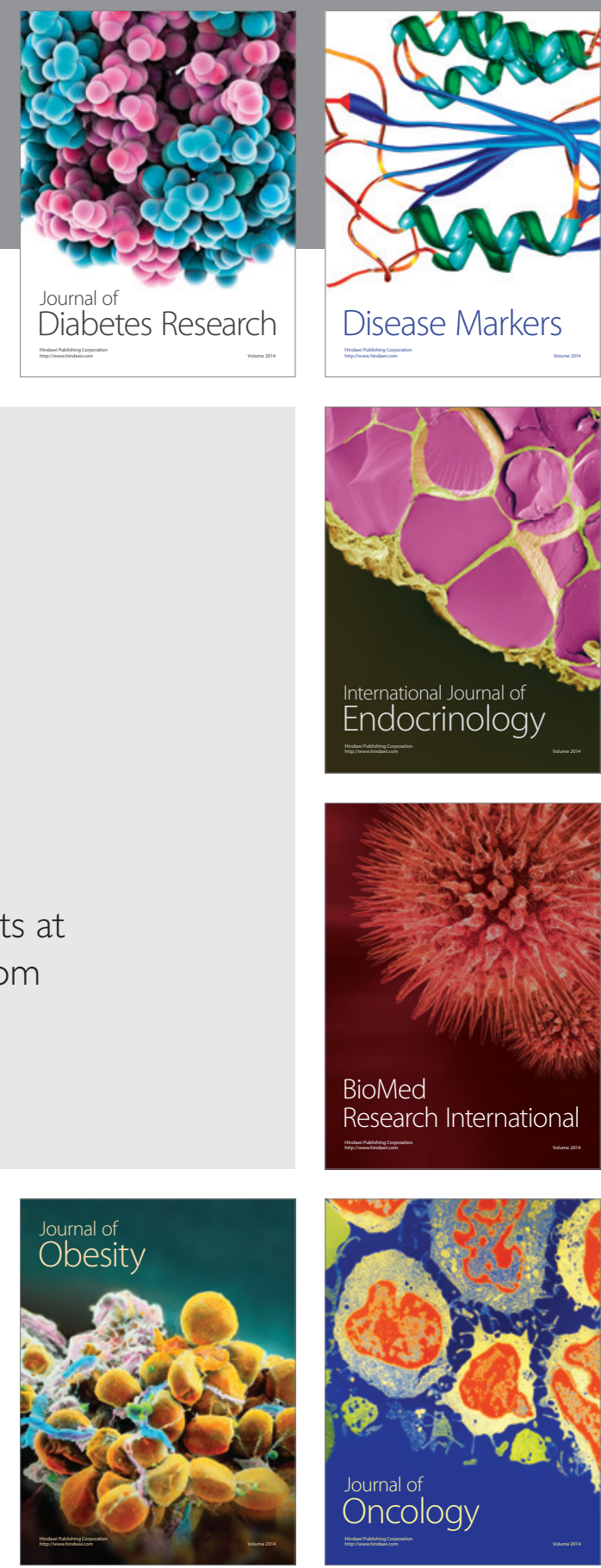

Disease Markers
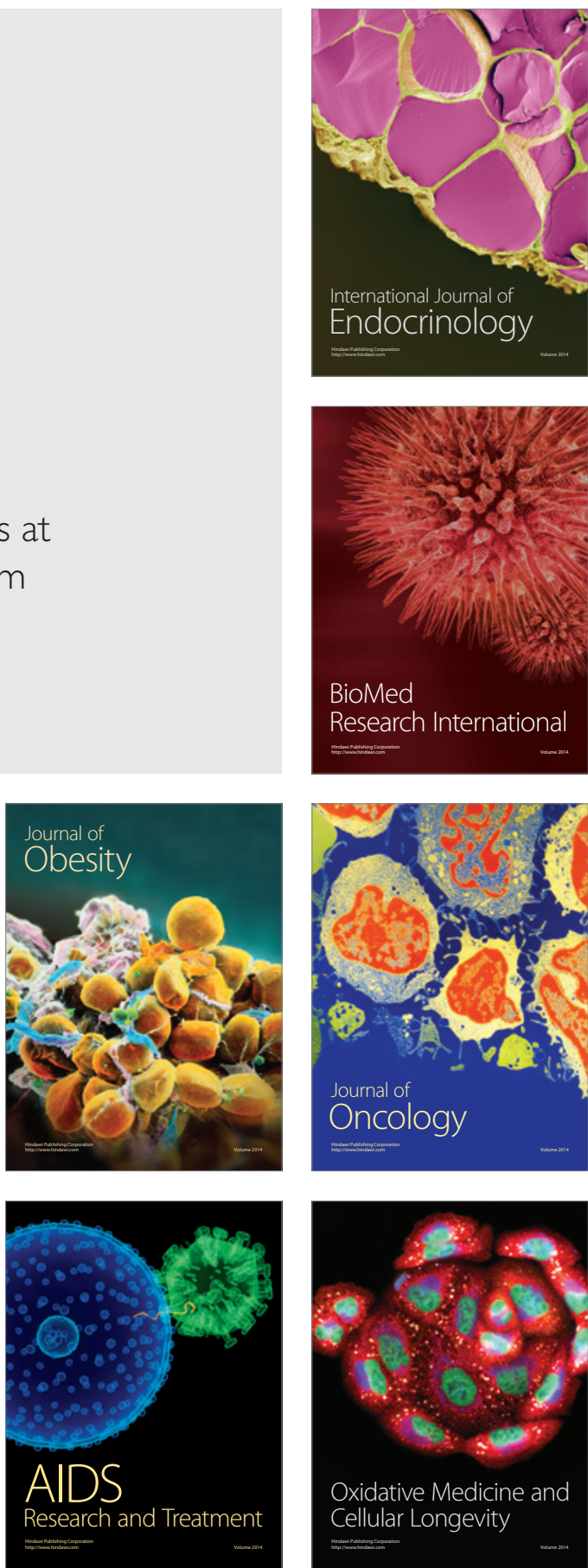